\title{
Emerging arboviruses in Quebec, Canada: assessing public health risk by serology in humans, horses and pet dogs
}

\author{
J. P. ROCHELEAU ${ }^{1} *$ P. MICHEL ${ }^{1,2}$, L. R. LINDSAY ${ }^{3}$, M. DREBOT ${ }^{3}$, \\ A. DIBERNARDO ${ }^{3}$, N.H OGDEN ${ }^{1,4}$, A. FORTIN ${ }^{5}$ AND J. ARSENAULT ${ }^{1,6}$ \\ ${ }^{1}$ Groupe de recherche en épidémiologie des zoonoses et santé publique, Faculté de médecine vétérinaire, \\ Université de Montréal, Saint-Hyacinthe, Québec, Canada \\ ${ }^{2}$ Office of the Chief Science Officer, Public Health Agency of Canada, Ottawa, Ontario, Canada \\ ${ }^{3}$ National Microbiology Laboratory, Public Health Agency of Canada, Winnipeg, Manitoba, Canada \\ ${ }^{4}$ National Microbiology Laboratory, Public Health Agency of Canada, Saint-Hyacinthe, Québec, Canada \\ ${ }^{5}$ Institut national de santé publique, Québec, Québec, Canada \\ ${ }^{6}$ Département de pathologie et microbiologie vétérinaire, Faculté de médecine vétérinaire, Université de Montréal, \\ Saint-Hyacinthe, Québec, Canada
}

Received 9 February 2017; Final revision 8 August 2017; Accepted 4 September 2017; first published online 28 September 2017

\section{SUMMARY}

Periodic outbreaks of West Nile virus (WNV), Eastern equine encephalitis virus (EEEV) and to a lesser extent, California serogroup viruses (CSGV), have been reported in parts of Canada in the last decade. This study was designed to provide a broad assessment of arboviral activity in Quebec, Canada, by conducting serological surveys for these arboviruses in 196 horses, 1442 dogs and 485 humans. Sera were screened by a competitive enzyme linked immunosorbent assay and positive samples confirmed by plaque reduction neutralisation tests. The percentage of seropositive samples was $83 \cdot 7 \%, 16 \cdot 5 \%, 7 \cdot 1 \%$ in horses, $18 \cdot 8 \%, 0 \cdot 6 \%, 0 \%$ in humans, $11 \cdot 7 \%$, $3 \cdot 1 \%, 0 \%$ in adult dogs and $2 \cdot 9 \%, 0 \cdot 3 \%, 0 \%$ in juvenile dogs for CSGV, WNV and EEEV, respectively. Serological results in horses and dogs appeared to provide a meaningful assessment of risk to public health posed by multiple arboviruses.

Key words: Arboviruses, California encephalitis virus, dogs, eastern equine, encephalomyelitis, horses, humans, public health, risk assessment, surveillance, West Nile virus.

\section{INTRODUCTION}

In the last two decades, various factors such as globalisation or ecological and climatic changes have driven the emergence of a number of arboviruses in different parts of the world [1]. Northern countries like Canada are no exception to this trend. West Nile virus (WNV) was introduced in Canada in 2001 [2], Eastern equine

\footnotetext{
* Author for correspondence: J. P. Rocheleau, Groupe de recherche en épidémiologie des zoonoses et santé publique, Faculté de médecine vétérinaire, Université de Montréal, 3200 rue Sicotte, Saint-Hyacinthe, QC, Canada J2S 2M2.

(Email: jean-philippe.rocheleau@umontreal.ca)
}

encephalitis virus (EEEV) is believed to have become endemic in locations in the Canadian provinces of Quebec and Ontario in the last decade [3, 4] and two California serogroup viruses (CSGV), Jamestown Canyon virus (JCV) and Snowshoe Hare virus (SHV), have recently shown evidence of emergence and increased recognition as human pathogens [5]. These viruses are amplified and maintained in transmission cycles involving wildlife reservoir hosts and many different species of mosquitoes that act, with varying degrees of competence, as vectors. All these viruses have the potential to cause moderate to severe encephalitis in humans by a similar pathogenic pathway [6]. 
Assessment of viral activity of emerging arboviruses is essential for the risk assessment for human populations, which is required to plan efficient, targeted preventive intervention strategies [7]. However, conducting a comprehensive assessment of arboviral activity in a given area is challenging and generally requires the joint analysis of different types of data [8-10]. These data include those from targeted entomological surveillance, active or passive surveillance for evidence of these agents in human or animal clinical cases, and serological surveillance in wild or domestic avian species [11-13]. Serological surveys have also been conducted in the past 15 years in domesticated animals such as horses, cats and dogs to assess if these species could act as sentinels for specific arboviruses [4, 14-16]. Results to-date suggest that serological monitoring in these animals could be a complementary approach for arbovirus surveillance and risk assessment. In this view, further seroprevalence data describing viral activity in humans and domestic species living within the same geographical area is needed to better articulate the possible relevance of systematically collecting data from domesticated animal populations for public health goals.

In the province of Quebec, Canada, surveillance for arboviruses has focused mainly on the collection and testing of mosquitoes for $\mathrm{WNV}$, for which a provincial public health surveillance program is currently in place $[17,18]$, and occasionally for other arboviruses like EEEV [19]. Although a few EEEV clinical cases in horses have been reported from Quebec since 2008 [4] and CSGV activity has been sporadically reported in this area since the mid-seventies [5], no human clinical cases of EEEV and only one laboratory confirmed human clinical case attributable to JCV have been reported in this province in the last decade [20]. The rarity or absence of laboratory confirmed clinical cases of EEEV and CSGV may be the result of physicians being unaware that diagnostic testing is available for these pathogens and as a result, outcomes from humans would significantly underestimate the level of arbovirus activity. In this context, this study aimed to provide an assessment of viral activity for WNV, CSGV and EEEV in southern Quebec by estimating the regional prevalence of antibodies to these arboviruses in humans, horses and dogs, with the perspective of evaluating whether serology in these two animal species provides meaningful information about public health risk for these arboviruses.

\section{MATERIAL AND METHODS}

\section{Study design and study area}

A cross-sectional study was conducted on horses, dogs and humans located within the same geographical region between 2012 and 2014. The study area encompassed five administrative regions in southern Quebec: Montreal, Laval, Montérégie, Lanaudière and Laurentides (Fig. 1), which were selected based on reported WNV and EEEV activity since 2008 [21, 22]. The study area had a human population of approximately $4 \cdot 7$ million people in 2011 [23].

\section{Sampling}

Horses

Blood samples from horses $>18$ months of age were collected in 2012 as described previously [4]. All horses resided within Montérégie, Lanaudière or Laurentides regions (Fig. 1) and none of the animals had been vaccinated against EEEV or WNV [4]. Sampling design was a convenience cluster sampling with barns as the primary sampling unit.

\section{Dogs}

Blood samples were collected from dogs between 27 May and 2 October 2013 by veterinarians or their technicians in veterinary clinics and hospitals distributed in the five selected regions (Fig. 1). Veterinary clinics and hospitals were identified based on a provincial registry and approximately 20 dogs were then selected by convenience by veterinary staff in each establishment. Dogs were categorized as 'adults' if they were born before 1 November 2012 and as 'juvenile' otherwise. These categories were chosen to account for possible exposure of dogs to mosquitoes before or only during the 2013 summer season when samples were collected from the animals. Veterinary staff was asked to sample adult dogs in June 2013 and to sample juvenile dogs regularly from July to October 2013. All dogs 4 months old or younger were excluded from sampling to avoid detecting antibodies from passive maternal immunity transfer [24].

\section{Humans}

Owners of sampled dogs were contacted by phone between February and May 2014 and asked to participate in the study. All adults living at the same address as one of the sampled dogs were asked to participate (Fig. 1). Blood samples were collected at home by a 

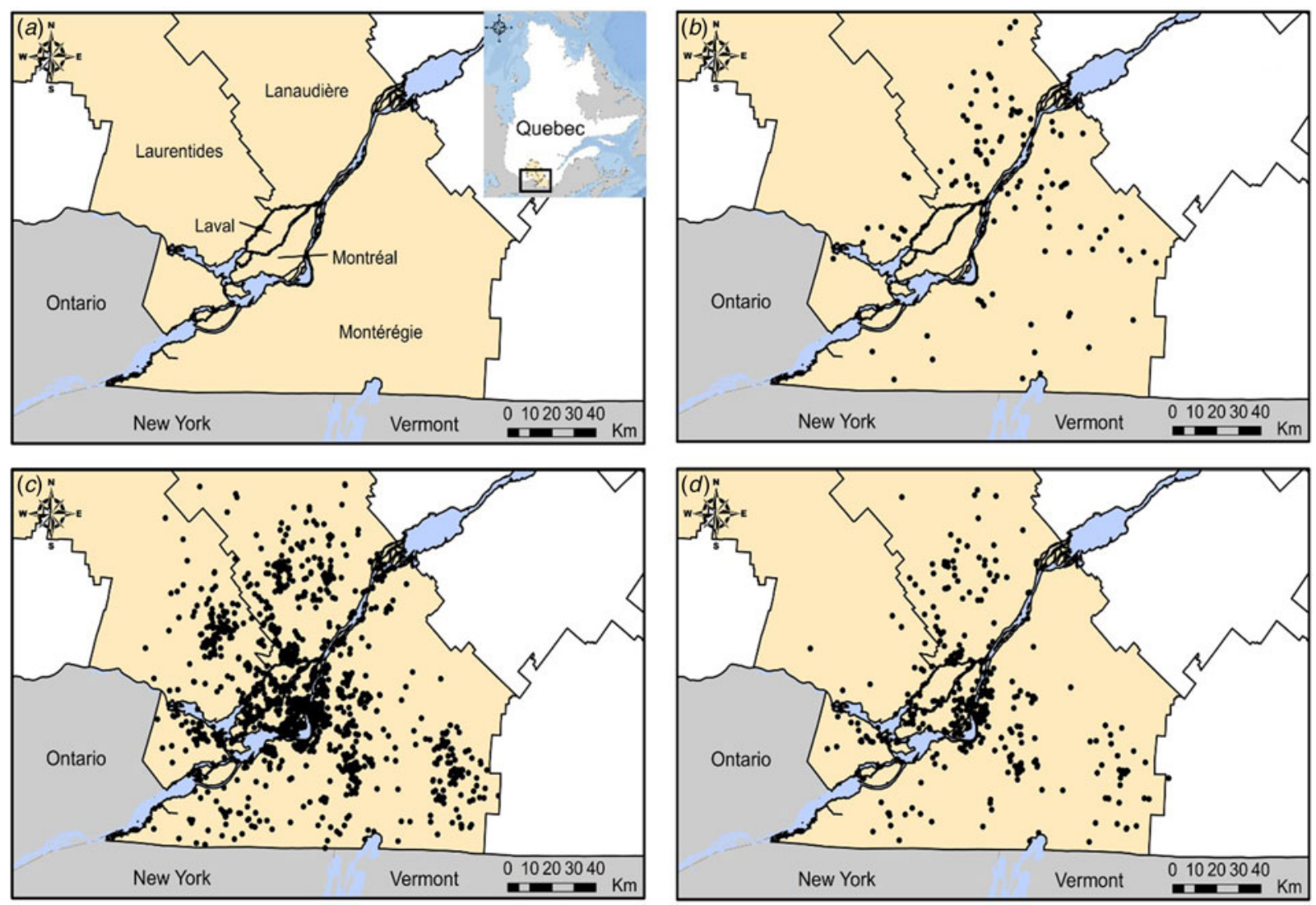

Fig. 1. The study area and geographical sampling distribution for serological surveys in horses, dogs and humans in five administrative regions of southern Quebec, 2012-2014. (a) Five administrative regions of Quebec where sampling was conducted; (b) geographical distribution of barns where horses were housed at the time of sampling in 2012; (c) geographical distribution of the place of residence of dogs at the time of sampling in 2013; $(d)$ geographical distribution of the place of residence of humans at the time of sampling in 2014.

nurse between 27 March and 10 June 2014 and all study participants provided informed consent under human research ethics approval (ethics approval number 13-130-CERES-D) from the Université de Montréal. The age of participants, by 10 year intervals, was collected upon sampling.

Human, equine, and canine blood samples were centrifuged for $10 \mathrm{~min}$ at $3000 \mathrm{rpm}$ and serum was removed, frozen at $-20^{\circ} \mathrm{C}$ and shipped to the National Microbiology Laboratory (NML) of the Public Health Agency of Canada for testing.

\section{Laboratory analyses}

All sera were tested for WNV and EEEV, but only a random subsample of sera were tested for CSGV as higher seroprevalences were expected. Subsamples of horses and humans were selected randomly. The dogs owned by all selected humans were then included for CSGV testing plus 88 additional dogs, which were randomly selected. Sera were first tested for antibodies to WNV, EEEV and CSGV by competitive enzyme linked immunosorbent assays (cELISA), except equine sera, which were tested directly by a plaque reduction neutralisation test (PRNT) for CSGV and EEEV antibodies. All sera that were positive by cELISA were subsequently tested by virus-specific PRNT. In all species and for all arboviruses studied, a positive PRNT result was required for classifying an animal as seropositive. The testing protocol chosen for each arbovirus in each species is summarized in Table 1.

\section{Viruses}

All viral antigens were produced in-house at the National Microbiology Laboratory, Public Health Agency of Canada, Winnipeg, Canada. WNV antigen was generated in suckling mice and prepared as $\beta$-propiolactone-inactivated sucrose-acetone extracts by the method of Clarke and Casals [25], for use in 
Table 1. Serological essays for California serogroup viruses, West Nile Virus and Eastern equine encephalitis virus in horses, dogs and humans from five administrative regions of Quebec, Canada, 2012-2014

\begin{tabular}{|c|c|c|c|c|}
\hline & \multirow{2}{*}{$\begin{array}{l}\text { Number of } \\
\text { sample tested }\end{array}$} & \multirow{2}{*}{$\begin{array}{l}\text { Years of } \\
\text { sampling }\end{array}$} & \multicolumn{2}{|l|}{ Test } \\
\hline & & & Screening & Confirmatory \\
\hline \multicolumn{5}{|l|}{ CSGV } \\
\hline Horse & 105 & 2012 & Not done & PRNT \\
\hline Dogs - juveniles & 170 & 2013 & cELISA* & PRNT \\
\hline Dogs - adults & 214 & 2013 & cELISA* & PRNT \\
\hline Humans & 400 & 2014 & cELISA* & $\mathrm{PRNT}^{\dagger}$ \\
\hline \multicolumn{5}{|l|}{ WNV } \\
\hline Horse & 196 & 2012 & cELISA* & PRNT \\
\hline Dogs - juveniles & 665 & 2013 & cELISA* & PRNT \\
\hline Dogs - adults & 777 & 2013 & cELISA* & PRNT \\
\hline Humans & 485 & 2014 & cELISA* & PRNT \\
\hline \multicolumn{5}{|l|}{ EEEV } \\
\hline Horse & 196 & 2012 & Not done & PRNT \\
\hline Dogs - juveniles & 665 & 2013 & cELISA* & Not done \\
\hline Dogs - adults & 777 & 2013 & cELISA* & Not done \\
\hline Humans & 485 & 2014 & cELISA* & Not done \\
\hline
\end{tabular}

CSGV, California serogroup viruses (Jamestown Canyon virus and Snowshoe hare virus only); WNV, West Nile virus; EEEV, Eastern Equine Encephalitis virus; cELISA, competitive enzyme linked immunosorbent assay; PRNT, plaque reduction neutralisation test - when used in combination with the cELISA, the PRNT was used to confirm all cELISA positive samples, except for CSGV in humans (see $\dagger$ ).

* Cut-off of $30 \%$ for positivity.

$\dagger$ PRNT processed on a subsample of 20 human cELISA seropositive samples to report adjusted percentages in humans;

$\$$ Because positive samples were not detected in the screening assay, PRNT were not performed on these samples.

the cELISA. The remaining viruses, EEEV, JCV and SHV were propagated on Vero E6 cells. Normal mouse brain antigen or Vero cell lysate was used as a control in all cELISAs.

\section{Monoclonal antibodies (MAbs)}

The panel of monoclonal antibodies used in this study consisted of MAB8152 (Millipore, Temecula, CA) and MAB7H2 (BioReliance, Rockville, MD), MAB2A2C-3 (Alphavirus group-specific conjugate, Centers for Disease Control and Prevention) and MAB10G5-4 (La Crosse encephalitis virus conjugate, Centers for Disease Control and Prevention). All MAbs detect E protein epitopes except MAB8152, which detects an NS1 epitope. Monoclonal antibodies for CSGV and EEEV were labelled with horseradish peroxidase (HRP) while WNV MAbs were unlabeled.

\section{cELISA}

Competitive ELISAs were based on the protocol of Blitvich et al. [26]. This assay was modified slightly for the detection of antibodies to $\mathrm{WNV}$, and served as the model for the development of in-house cELISAs for antibodies to EEEV and CSGV. Briefly, 96 well microtitre plates (Dynatech Immulon H2B, VWR, Mississauga, ON, Cat. \# 62402-972) were coated overnight with suckling mouse brain or Vero E6 cell-derived viral antigen and control antigen diluted in carbonate-bicarbonate buffer, $\mathrm{pH} 9 \cdot 6$. Wells were blocked with PBS containing 5\% skim milk, washed, and test sera diluted 1:10 for WNV and EEEV or 1:20 for CSGV cELISA, were then added. Virus-specific conjugated MAbs MAB2A2C-3/HRP and 10G5-4-HRP targeting EEEV and CSGV, respectively, were then applied to each well. For WNV antibody detection, unconjugated MAbs MAB8152 and MAB7H2 were used, followed by a peroxidase-labelled anti-mouse IgM (Mandel Scientific, Guelph, ON, (KPL), Cat. \# 074-1803) and anti-mouse IgG (Fisher Scientific, (Pierce), Cat. \# 31430) conjugates, respectively. A chromogenic substrate, 3,3',5,5'-tetramethylbenzidine (SureBlue TMB, Mandel (KPL)), Cat. \# 52-00-00) was added for colour development, which was subsequently stopped by the addition of $1 \mathrm{~N}$ sulphuric acid. The optical 
density was read at $450 \mathrm{~nm}$ and percent inhibition calculated. Samples which inhibited binding of the MAbs by $\geqslant 30 \%$ were considered positive.

\section{PRNTS}

PRNTs on serum specimens were performed as previously described [27]. Briefly, a constant number of plaque forming units (PFU) of WNV, EEEV or CSGV (SHV and JCV), was incubated with various dilutions of test sera for $1 \mathrm{~h}$ at $37^{\circ} \mathrm{C}$ with $5 \% \mathrm{CO}_{2}$ to allow virus neutralization. An aliquot of this mixture was then added to 6-well plates containing confluent Vero E6 cell monolayers and further incubated for $1 \mathrm{~h}$ at $37^{\circ}$ $\mathrm{C}$ at which point a nutrient agar overlay was added. The plates were incubated at $37^{\circ} \mathrm{C}$ with $5 \% \mathrm{CO}_{2}$ for approximately 3 days, and another overlay containing neutral red as a vital stain was added to visualize plaque formation. Serum samples inhibiting at least $90 \%$ of possible plaque formation relative to virus controls were deemed positive for viral antibodies. The highest serum dilution with a plaque reduction of at least $90 \%$ was defined as the titration endpoint. The PRNT results were considered positive if neutralizing antibody titre was greater than or equal to $1: 20$. Since some serological cross reactivity between CSG viruses can occur, specific virus exposures were assigned if fourfold or greater neutralization titres to either JCV or SHV were identified upon completion of PRNT assays.

\section{Data analyses}

All study subjects were geolocated according to their street address with GeoPinpoint software (DMTI Spatial Inc, ON, Canada) and mapped in ArcGIS 10.2.2 (ESRI, Redlands, CA, USA). The proportion of males and females and the median age, along with its interquartile range (IQR), were reported for each species. IQR were not given for age in humans considering the categorical nature of this variable. The percentages of seropositive samples were reported for each virus. Regional seroprevalence estimates and 95\% confidence intervals were estimated with standard errors adjusted for clustered sampling (household or barns) using the surveyfreq procedure in SAS $9 \cdot 4$.

\section{RESULTS}

Serum samples were taken from 196 unvaccinated horses housed within 92 barns as described in Rocheleau et al. [4]. For dogs, a total of 260 veterinary clinics and hospitals were identified in the study area. The owners of these 260 clinics were reached by phone. A total of 47 clinics were excluded because their dog-owning clientele was too small to allow participation in the study. Among the 213 remaining veterinary clinics and hospitals, $89(42 \%)$ agreed to participate. A total of 1627 dogs, from 1582 different households, were sampled. Age and address were available for 1442 of the 1627 dogs. Of these 1442 dogs, 777 were adult dogs sampled in June and the first week of July 2013, and 665 were juvenile dogs sampled from late June to September 2013. A total of 485 humans, living in 374 households, were sampled in 2014.

The geographical distribution of the study subjects is presented in Figure 1. Median age of study subjects was $9 \cdot 0$ years old for horses (IQR $=6 \cdot 0), 4 \cdot 6$ years old for adult dogs $(\mathrm{IQR}=4 \cdot 8), 0.49$ years old for juvenile $\operatorname{dogs}(\mathrm{IQR}=0 \cdot 18)$ and approximately 45 years old for humans. A third of the humans that were tested were males while approximately $50 \%$ of dogs and horses from which samples were collected were males.

The percentage of seropositive samples was $83.7 \%$, $16 \cdot 5 \%, 7 \cdot 1 \%$ in horses, $18 \cdot 8 \%, 0 \cdot 6 \%, 0 \%$ in humans, $11 \cdot 7 \%, 3 \cdot 1 \%, 0 \%$ in adult dogs and $2 \cdot 9 \%, 0 \cdot 3 \%, 0 \%$ in juvenile dogs for CSGV, WNV and EEEV, respectively. For CSGV, subsamples of 105 horses, 384 dogs and 400 humans were randomly selected for testing. For CSGV in humans, among the 20 sera that were positive on the cELISA and which were tested by PRNT, four $(20 \%)$ were negative, seven $(35 \%)$ were specifically assigned to JCV, four (20\%) were assigned to SHV and five $(25 \%)$ were positive to both JCV and SHV and could not be assigned specifically to one of these two viruses. For CSGV in dogs, among the 32 sera that were positive on the cELISA, $5(16 \%)$ were negative on PRNT, 14 (44\%) were specifically assigned to $\mathrm{JCV}$, one $(3 \%)$ was assigned to SHV and $12(37 \%)$ were positive to both JCV and SHV. In both humans and dogs, a positive PRNT for both SHV and JCV could indicate either exposure to both viruses or cross-reaction between the two viruses. Given the high percentage of CSGV positive sera that could not be assigned to a specific virus in humans and dogs, seroprevalence results were reported jointly for JCV and SHV. Regional seroprevalence estimates for WNV, CSGV and EEEV are given in Table 2 for each species in which antibodies were detected. In humans, seroprevalence estimates were higher for $\mathrm{CSGV}$ than for $\mathrm{WNV}$ in all regions, without overlapping confidence intervals for 
Table 2. Regional seroprevalence estimates for antibodies to West Nile virus and California serogroup viruses in horses, dogs and humans from southern Quebec, 2012-2014

\begin{tabular}{|c|c|c|c|c|c|c|c|c|c|c|}
\hline & \multicolumn{2}{|c|}{ Lanaudière } & \multicolumn{2}{|c|}{ Laurentides } & \multicolumn{2}{|c|}{ Montérégie } & \multicolumn{2}{|c|}{ Laval } & \multicolumn{2}{|c|}{ Montreal } \\
\hline & $n$ & $\mathrm{SP} \%(95 \% \mathrm{CI})$ & $n$ & $\mathrm{SP} \%(95 \% \mathrm{CI})$ & $n$ & $\mathrm{SP} \%(95 \% \mathrm{CI})$ & $n$ & $\mathrm{SP} \%(95 \% \mathrm{CI})$ & $n$ & $\mathrm{SP} \%(95 \% \mathrm{CI})$ \\
\hline \multicolumn{11}{|l|}{ WNV } \\
\hline Horse & 72 & $11 \cdot 9(5 \cdot 3-22 \cdot 2)$ & 18 & $16 \cdot 7(3 \cdot 6-41 \cdot 4)$ & 104 & $20 \cdot 2(12 \cdot 5-27 \cdot 9)$ & NA & & NA & \\
\hline Juv. Dogs & 149 & $0 \cdot 7(0 \cdot 02-3 \cdot 7)$ & 66 & $0 \cdot 0(0 \cdot 0-5 \cdot 4)$ & 331 & $0 \cdot 3(0 \cdot 01-1 \cdot 7)$ & 26 & $0 \cdot 0(0 \cdot 0-13 \cdot 2)$ & 88 & $0 \cdot 0(0 \cdot 0-4 \cdot 1)$ \\
\hline Adult dogs & 109 & $2 \cdot 8(0 \cdot 6-7 \cdot 8)$ & 68 & $5.9(1 \cdot 6-14 \cdot 4)$ & 372 & $3 \cdot 2(1 \cdot 7-5 \cdot 6)$ & 36 & $2 \cdot 8(0 \cdot 07-14 \cdot 5)$ & 182 & $2 \cdot 2(0 \cdot 6-5 \cdot 5)$ \\
\hline Humans & 112 & $0 \cdot 0(0 \cdot 0-3 \cdot 2)$ & 19 & $5 \cdot 3(0 \cdot 1-26 \cdot 0)$ & 256 & $0 \cdot 8(0 \cdot 09-2 \cdot 8)$ & 11 & $0 \cdot 0(0 \cdot 0-28 \cdot 5)$ & 85 & $0 \cdot 0(0 \cdot 0-4 \cdot 25)$ \\
\hline \multicolumn{11}{|l|}{ CSGV } \\
\hline Horse & 48 & $77 \cdot 1(62 \cdot 7-88 \cdot 0)$ & NA & & 56 & $89 \cdot 3(78 \cdot 1-96 \cdot 0)$ & NA & & NA & \\
\hline Juv. dogs & 15 & $9 \cdot 8(2 \cdot 7-23 \cdot 1)$ & 41 & $0 \cdot 0(0 \cdot 0-21 \cdot 8)$ & 85 & $1 \cdot 2(0 \cdot 03-6 \cdot 4)$ & 6 & $0 \cdot 0(0 \cdot 0-45 \cdot 9)$ & 23 & $0 \cdot 0(0 \cdot 0-14 \cdot 8)$ \\
\hline Adult dogs & 30 & $20 \cdot 0(7 \cdot 7-38 \cdot 6)$ & 17 & $5 \cdot 9(0 \cdot 2-28 \cdot 7)$ & 113 & $12 \cdot 4(7 \cdot 9-19 \cdot 9)$ & 3 & $0 \cdot 0(0 \cdot 0-70 \cdot 8)$ & 46 & $6 \cdot 5(1 \cdot 4-17 \cdot 9)$ \\
\hline Humans & 89 & $32 \cdot 6(23 \cdot 0-43 \cdot 3)$ & 16 & $31 \cdot 2(11 \cdot 0-58 \cdot 7)$ & 212 & $21 \cdot 2(15 \cdot 9-27 \cdot 4)$ & 10 & $10 \cdot 0(0 \cdot 3-44 \cdot 5)$ & 71 & $15 \cdot 5(8 \cdot 0-26 \cdot 0)$ \\
\hline \multicolumn{11}{|c|}{ 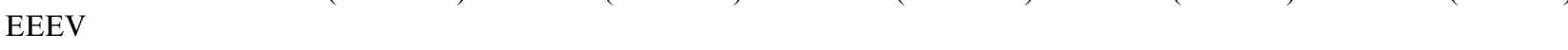 } \\
\hline Horses & 72 & $11 \cdot 8(2 \cdot 6-20 \cdot 9)$ & 18 & $0 \cdot 0(0 \cdot 0-19 \cdot 0)$ & 104 & $6 \cdot 7(1 \cdot 5-12 \cdot 0)$ & NA & & NA & \\
\hline
\end{tabular}

$n$, regional sample size; SP, seroprevalence; WNV, West Nile virus; CSGV, California serogroup viruses (Jamestown Canyon virus and Snowshoe hare virus only); NA, no estimate available since no horse were tested; Juv, juvenile.

the regions of Lanaudière, Montérégie and Montréal where sample sizes were larger. In all regions where horses were sampled, we observed higher seroprevalence estimates in horses than in other species for all the arboviruses studied. We noted higher seroprevalence estimates for WNV for adult dogs and humans in the region of Laurentides compared with the other regions.

\section{DISCUSSION}

The seroprevalence estimates in horses, adult dogs, and humans provided evidence of sustained arboviral activity in the southern region of Quebec. In addition, these estimates in juvenile dogs indicated that, at least for CSGV and WNV, virus transmission occurred during the summer of 2013.

In all species, the regional seroprevalences for CSGV were high and generally higher than for WNV. Similar seroprevalences for JCV and SHV were previously reported in animals and humans from other locations in Canada. Goff et al. [28] reported a seroprevalence of $76 \%$ in horses from Newfoundland in 2008 and Artsob [29] reported seroprevalences ranging from $0.5 \%$ to $32 \%$ in humans from different locations across Canada between 1970 and 1980. When considered along with the reported ratios of asymptomatic to neuro-invasive infections for each arbovirus in humans (approximately 1500:1 for CSGV and from 150:1 to 250:1 for WNV [5, 30]), these findings suggest that physicians should include CSGV as well as WNV in their differential diagnosis of acute human encephalitis even though the risk of developing clinical signs when infected by CSGV seems to be low [5]. Relatively to other regions, seroprevalence estimates observed for the region of Lanaudière suggest that this region has ecological characteristics that favour CSGV and EEEV transmission.

The high seroprevalence in horses reported for EEEV, WNV and CSGV suggests that horses are highly exposed to various arboviruses and that serology in horses may be a very sensitive indicator of arboviral activity in a given area. In this regard, monitoring seroconversion or assessing seroprevalence in horses on a regular basis could allow for early detection or trend assessment for various emerging arboviral threats even for arboviruses to which horses are not clinically sensitive. Although regional seroprevalence estimates in domestic dogs were much lower than in horses, dogs also provided a sensitive indication of past or ongoing arboviral activity. Previous serosurveys for WNV in dogs suggested that dogs could act as sensitive indicators of WNV activity [14]. The current results extend this notion to CSGV.

Two findings were made after exploration of our seroprevalence estimates. On the one hand, for the three arboviruses, the proportion of seropositive subjects was generally lower in young dogs, intermediate in adult dogs and humans and higher in horses. On the other hand, in all species, the proportion of seropositive subjects was generally higher for CSGV, 
intermediate for WNV and low for EEEV. Thus, the risk of being exposed to one arbovirus compared with another seems to be relatively consistent between species. Although these findings would need to be confirmed with larger and randomly selected samples, we may hypothesise (1) that exposure to mosquito bites in general varies greatly across species but (2) that horses, dogs and humans have similar relative contact rates with each of the different vector species associated with arboviral transmission in Quebec. This second hypothesis is consistent with the fact that each of the arboviruses studied is transmitted by specific competent vector species (mainly vectors of genus Aedes and Ochlerotatus for CSGV [5, 28], Culiseta for EEEV [4] and Culex for WNV [2]). Therefore, contact rates with infected Aedes or Ochlerotatus vectors seem relatively high in horses, dogs and humans while contact rates with infected Culex or Culiseta vectors are probably relatively low in the three animal species. Similar feeding preferences of mosquitoes towards horses, dogs, and humans have previously been reported [31-33].

Exposure to mosquito bites may be related to various factors such as the time spent outside daily, the breed characteristics or natural or acquired protective behaviours. Among these factors, the time spent outside daily may be a key factor contributing to the differences between seroprevalence estimates in horses, dogs and humans. For example, in a serosurvey of companion animals conducted in 2002 in Louisiana, Kile et al. reported a 19-fold greater probability of seropositivity for WNV in outdoor-only family dogs compared with indoor family dogs [16]. While this factor can bias seroprevalence estimates in seroprevalence studies, it may represent an advantage in other contexts. For example, selecting animals based on their outdoor exposure could improve the sensitivity of a surveillance system based on serology. In addition to the factors mentioned, some differences in seroprevalence estimates between species may relate to the fact that temporal opportunities for exposure to the different arboviruses were not the same among viruses and target species. For example, CSGV have been circulating in Canada for centuries if not longer $[29,34]$, and antibodies to these viruses would be expected to persist for many years in naturally infected humans [35]. Since the humans surveyed were a median age of approximately 45 years old and could have been exposed to CSGV at any time point over their lifetime and since the median age of sampled dogs was only 4.6 years old, the timeframe for possible exposure to arboviruses was much shorter in dogs than in humans. In addition, WNV was only introduced into Quebec in 2001, with the largest WNV outbreak in humans reported in 2012. This outbreak gave rise to 134 of the $268(50 \%)$ human clinical cases reported from 2002 to 2014 [36]. Most of the adult dogs that were sampled were born at the onset of the 2012 WNV outbreak.

Although the sensitivity and specificity of the serological tests that we used in this study were not formally assessed, the number of false positive reactions is expected to be low for all arboviruses studied for the following reasons. The MAbs used for WNV ELISA were previously reported to be highly specific for WNV with no cross-reaction with Saint-Louis encephalitis virus, which is the only other mosquitoborne flavivirus reported in Canada so far [26]. Moreover, the known geographical distribution of other alphaviruses such as Western or Venezuelan equine encephalitis does not extend to the province of Quebec, limiting the possibility of cross-reactions directed against EEEV. Finally, in addition to JCV and SHV, Trivitattus virus is the only orthobunyavirus reported in Canada to date and was only identified in three Ontario mosquitoes in 1976 [29], suggesting a low probability of cross-reactions between JCV/SHV and other CSGV in the sampled populations. The reported sensitivity for the WNV cELISA is usually superior to $90 \%$ [26, 27]. However, no sensitivity estimate is currently available for EEEV or CSGV. Hence, given the (1) probable imperfect sensitivity of the cELISA, (2) use of PRNT as a confirmatory test for all samples seropositive on cELISA and (3) high specificity expected for PRNT [37], seroprevalence estimates reported in this study should be interpreted as conservative estimates.

As samples could not be randomized in any of the studied populations, caution is required about potential biases in seroprevalence estimates if the aim is to extrapolate these seroprevalence estimates to the whole population of humans, dogs or horses from southern Quebec. For example, in the context of this study, differential outdoor exposure in males and females may have biased seroprevalence estimates in humans given that males were underrepresented in the sample. However, the seroprevalence of antibodies to WNV in humans in this study was relatively comparable with the results from a serosurvey conducted in southern Ontario after the $2002 \mathrm{WNV}$ outbreak in humans (3.1\% [38]) and helps support the notion that humans are rarely bitten by WNV-infected mosquitoes in eastern 
Canada. Moreover, as discussed in Rocheleau et al. [4], there are specific limitations to the use of serology in horses pertaining to the fact that clear information on vaccination status must be obtained in each horse for adequately interpreting WNV and EEEV serological results. However, to our knowledge, current vaccination protocols have no impact on serological status for CSGV since no vaccines are currently available for these mosquito-borne bunyaviruses.

This study was conducted from a public health perspective. Evidence for transmission of multiple arboviruses was detected, and the approach was designed to target easily accessible animals with the aim to compare results with serological data in humans from a similar geographical and temporal frame. Operational and ethical considerations linked with performing epidemiological assessments involving human subjects may lead to more challenging study implementation than one involving non-human subjects. Assuming a reliable correspondence between animal exposures for public health, studies in selected domestic animal species may thus lead to an efficient approach for building knowledge, exploring emergence and providing an early assessment of the regional risk posed by arboviruses to human populations. As CSGV, WNV and EEEV are not the only arboviruses with potential to emerge or re-emerge in Canada and as serological surveillance in the selected animal species seems to be adaptable to various arboviruses, this approach might eventually allow for surveillance of multiple arboviruses based on readily available samples from the veterinary network.

\section{ACKNOWLEDGEMENTS}

We thank all veterinarians and animal health technicians who contributed to sampling and all participants involved in this study. We also thank Caroline Doré who coordinated the human sampling. We also thank Krisitina Dimitrova and Nicole Barairo at the NML for performing some of the serological assays. This study was funded by the Public Health Agency of Canada.

\section{DECLARATION OF INTEREST}

None.

\section{ETHICAL STANDARDS}

The human study protocol was approved by the Committee for ethical health research of the Université de Montréal (protocol 13-130-CERES-D) and all procedures on animals were approved by the Committee for ethical animal use of the same institution (protocols 12-Rech-1638 and 12-Rech-1698). The authors assert that all procedures contributing to this work comply with the ethical standards of the relevant national and institutional committees on human experimentation and with the Helsinki Declaration of 1975 , as revised in 2008 and with the ethical standards of the relevant national and institutional guides on the care and use of animals in research.

\section{REFERENCES}

1. Weaver SC, Reisen WK. Present and future arboviral threats. Antiviral Research 2010; 85: 328-345.

2. Komar N. West Nile virus: epidemiology and ecology in North America. Advances in Virus Research 2003; 61: $185-234$

3. Armstrong PM, Andreadis TG. Eastern equine encephalitis virus - old enemy, new threat. New England Journal of Medicine 2013; 368: 1670-1673.

4. Rocheleau JP, et al. Eastern equine encephalitis virus: high seroprevalence in horses from Southern Quebec, Canada, 2012. Vector Borne and Zoonotic Diseases 2013; 13: 712-718.

5. Drebot MA. Emerging mosquito-borne bunyaviruses in Canada. Canada Communicable Disease Report 2015; 41: 117-123.

6. Kuhn JH, Peters CJ. Arthropod-borne and rodentborne virus infections. In: Kasper $\mathrm{D}$, et al., eds. Harrison's Principles of Internal Medicine, 19e. New York, NY: McGraw-Hill, 2014 (http://accessmedicine. mhmedical.com/content.aspx?bookid=1130\&sectionid= 79739575). Accessed 21 September 2017.

7. Sorvillo F, Shafir S. Control of microbial threats: population surveillance, vaccine studies, and the microbiological laboratory. In: Detels R, et al., eds. Oxford Textbook of Public Health. New York: Oxford University Press, 2009, pp. 858-869.

8. Government of Canada. Surveillance of West Nile virus (http://healthycanadians.gc.ca/diseases-conditions-malad ies-affections/disease-maladie/west-nile-nil-occidental/sur veillance-eng.php). Accessed 13 February 2016.

9. Centers for Disease Control and Prevention. Guidelines for arboviruses surveillance programs in the United States (http://www.cdc.gov/ncezid/dvbd/pdf/arboguid_ 508.pdf). Accessed 3 October 2015.

10. Ontario Agency for Health Protection and Promotion (Public Health Ontario). Eastern equine encephalitis: history and enhanced surveillance in Ontario (http:// www.publichealthontario.ca/en/eRepository/Eastern Equine_Encephalitis_Virus_Report_2014.pdf). Accessed 17 January 2015.

11. Calisher CH, et al. Relevance of detection of immunoglobulin $\mathrm{M}$ antibody response in birds used for arbovirus surveillance. Journal of Clinical Microbiology 1986; 24: 770-774. 
12. Crans WJ. Failure of chickens to act as sentinels during an epizootic of eastern equine encephalitis in southern New Jersey, USA. Journal of Medical Entomology 1986; 23: 626-629.

13. Morris CD, et al. Comparison of chickens and pheasants as sentinels for eastern equine encephalitis and St. Louis encephalitis viruses in Florida. Journal of the American Mosquito Control Association 1994; 10: 545-548.

14. Resnick MP, et al. Juvenile dogs as potential sentinels for West Nile virus surveillance. Zoonoses and Public Health 2008; 55: 443-447.

15. Shimoda H, et al. Dogs as sentinels for human infection with Japanese encephalitis virus. Emerging Infectious Diseases 2010; 16: 1137-1139.

16. Kile JC, et al. Serologic survey of cats and dogs during an epidemic of West Nile virus infection in humans. Journal of the American Veterinary Medical Association 2005; 226: 1349-1353.

17. National Public Health Institute of Quebec. Strategic impact assessment of the government response plan for public health protection against West Nile virus Main Report (https://www.inspq.qc.ca/pdf/publications/687_Rapport_principal_EIS.pdf). Accessed 23 March 2015.

18. National Public Health Institute of Quebec. The risk of West Nile Virus in Quebec and interventions prioritized in 2013 (http://www.inspq.qc.ca/pdf/publications/1629_ RisqueVNOQcIntervPrivil2013.pdf). Accessed 7 July 2015.

19. Vincent C, et al. Surveillance of eastern equine encephalomyelitis in Quebec. Proceedings of the 2011 Conference Event detection and surveillance. St-Hyacinthe: Canadian Association of Veterinary Epidemiology and Preventive Medicine, 2011, p. 68.

20. National Public Health Institute of Quebec. 2011-2012 Activity report of the Quebec public health laboratory (https://www.inspq.qc.ca/publications/1595). Accessed 15 April 2015.

21. Quebec Ministry of Agriculture Fisheries and Food. Eastern equine encephalomyelitis (http://www.mapaq. gouv.qc.ca/fr/Productions/santeanimale/maladies/transmissibleshumain/Pages/encephalomyeliteequinedelest. aspx). Accessed 5 June 2015.

22. Quebec Ministry of Health and Social Services. West Nile Virus - Cases in Humans (http://www.msss.gouv. qc.ca/sujets/santepub/environnement/index.php?id=127, 156,0,0,1,0). Accessed 29 July 2015.

23. Statistics Canada. Census Profile 2011 - Health regions (December 2013) (http://www12.statcan.gc.ca/censusrecensement/2011/dp-pd/prof/details/download-telecharger/comprehensive/comp-csv-tab-dwnld-tlchrgr.cfm?Lang =E\#tabs2011). Accessed 11 December 2015.

24. Chappuis G. Neonatal immunity and immunisation in early age: lessons from veterinary medicine. Vaccine 1998; 16: 1468-1472.
25. Clarke DH, Casals J. Techniques for hemagglutination and hemagglutination-inhibition with arthropod-borne viruses. American Journal of Tropical Medicine and Hygiene 1958; 7: 561-573.

26. Blitvich BJ, et al. Epitope-blocking enzyme-linked immunosorbent assays for the detection of serum antibodies to West Nile virus in multiple avian species. Journal of Clinical Microbiology 2003; 41: 1041-1047.

27. Beaty BJ, Calisher CH, Shope RS. Arboviruses. In: Schmidt NJ, Emmons RW, eds. Diagnostic Procedures for Viral, Rickettsial and Chlamydial Infections. Washington: American Public Health Association, 1989, pp. 797-856.

28. Goff G, Whitney H, Drebot MA. Roles of host species, geographic separation, and isolation in the seroprevalence of Jamestown Canyon and snowshoe hare viruses in Newfoundland. Applied and Environmental Microbiology 2012; 78: 6734-6740.

29. Artsob H. Distribution of California serogroup viruses and virus infections in Canada. Progress in Clinical and Biological Research 1983; 123: 277-290.

30. Petersen LR, Brault AC, Nasci RS. West Nile virus: review of the literature. Journal of the American Medical Association 2013; 310: 308-315.

31. Molaei G, et al. Host feeding patterns of Culex mosquitoes and West Nile virus transmission, northeastern United States. Emerging Infectious Diseases 2006; 12: 468-474.

32. Molaei G, et al. Molecular identification of blood-meal sources in Culiseta melanura and Culiseta morsitans from an endemic focus of eastern equine encephalitis virus in New York. The American Journal of Tropical Medicine and Hygiene 2006; 75: 1140-1147.

33. Richards SL, et al. Host-feeding patterns of Aedes albopictus (Diptera: Culicidae) in relation to availability of human and domestic animals in suburban landscapes of central North Carolina. Journal of Medical Entomology 2006; 43: 543-551.

34. Ulloa A, et al. Serologic survey of domestic animals for zoonotic arbovirus infections in the Lacandon Forest region of Chiapas, Mexico. Vector Borne and Zoonotic Diseases 2003; 3: 3-9.

35. De Filette M, et al. Recent progress in West Nile virus diagnosis and vaccination. Veterinary Research 2012; 43: 16 .

36. National Public Health Institute of Quebec. Monitoring infection by West Nile virus in Quebec - 2014 Season (https://www.inspq.qc.ca/pdf/publications/2030_surveillance_infection_virus_nil.pdf). Accessed 15 April 2016.

37. World Organisation for Animal Health. Manual of diagnostic tests and vaccines for terrestrial animals, 2015 (http://www.oie.int/international-standard-setting/ terrestrial-manual/). Accessed 15 April 2016.

38. Loeb M, et al. Protective behavior and West Nile virus risk. Emerging Infectious Diseases 2005; 11: 1433-1436. 